Check for updates

Cite this: RSC Adv., 2017, 7, 47190

Received 1st June 2017

Accepted 1st October 2017

DOI: $10.1039 / \mathrm{c} 7 \mathrm{ra06125k}$

rsc.li/rsc-advances

\section{Vibrational characteristics of DNA nanostructures obtained through a mass-weighted chemical elastic network model $\uparrow$}

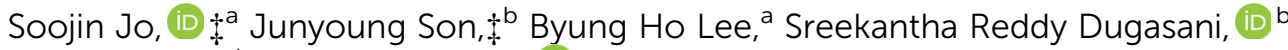 \\ Sung Ha Park ${ }^{\star b}$ and Moon Ki Kim (ID *a
}

Using the programmable and self-assembly characteristics of DNA, various DNA nanostructures have been designed and synthesized for specific applications, such as nanomachinery and chemical/biological sensors. Although their physical features and feasibility in engineering applications can be conjectured using experimental techniques such as atomic force microscopy and Raman spectroscopy, their vibration characteristics at low frequency states, which are the most dominant factors that determine their structural functions, are difficult to observe experimentally because it is almost impossible to capture the real-time atomic motion of DNA nanostructures. Here, we propose a novel method to elucidate the vibration characteristics of DNA nanostructures in atomic detail using a normal mode analysis based on a mass-weighted chemical elastic network model (MWCENM). Because the MWCENM is a precise method for modeling molecular structures that considers both chemical bond information and inertia effects, it can calculate both vibration frequencies and the corresponding mode shapes in atomic detail. In terms of vibration frequencies, our simulation results show good agreement, within an error deviation of $4.0 \%$, with experimental data measured by Raman spectroscopy. Therefore, the proposed theoretical approach is a feasible method for understanding DNA nanostructure's vibration characteristics, including both frequencies and mode shapes, in atomic detail, adding to the molecular fingerprint provided by the conventional Raman spectrum.

\section{Introduction}

For the past 30 years, DNA molecules have been considered one of the most promising building blocks for various dimensional nanostructures due to the programmability of their base sequences. ${ }^{1-7}$ Recently, DNA nanostructures have been used in many different applications in the fields of science, engineering, and nanobiotechnology. ${ }^{8-10}$ To build useful DNA nanostructures for specific applications, researchers need to statically and dynamically verify their engineering feasibility in terms of structural stability and vibrational characteristics. In a previous study, we used vibrational mode analysis to explain the basic self-assembly mechanism for the formation of circular DNA tubes made of planar double-crossover (DX) DNA tiles. ${ }^{11}$

Although certain physical characteristics are difficult to observe experimentally due to the measuring limitations of the

${ }^{a}$ School of Mechanical Engineering, Sungkyunkwan University, Suwon, 16419, Korea. E-mail:mkkim@me.skku.ac.kr

${ }^{b}$ Department of Physics, Sungkyunkwan University, Suwon, 16419, Korea. E-mail: sunghapark@skku.edu

$\dagger$ Electronic supplementary information (ESI) available. See DOI: 10.1039/c7ra06125k

\$ These authors contributed equally. apparatus or sample preparation, simulations can easily provide significant insights about physical properties. For instance, atomic vibration at low frequency (under $300 \mathrm{~cm}^{-1}$ ), which is of significant importance for understanding biological functionality and optimizing synthesis, is hard to measure experimentally. However, by using simulation, mode shapes from collective motion to local vibration can be analyzed at atomic level as shown in Fig. 1. In this study, we (i) suggest a way to sample atoms in DNA molecules for applying a massweighted chemical elastic network model (MWCENM), (ii) propose an intrinsic frequency analysis method based on the MWCENM, (iii) measure Raman spectrum of rectangular-shape DNA DX tiles, and (iv) validate the reliability of the proposed frequency analysis method by comparing its results with experimental data.

\section{Materials and methods}

\section{Mass-weighted chemical elastic network model (MWCENM)}

In the traditional elastic network model (ENM), representative atoms are modeled as unit point masses and their interaction is represented by a uniform spring network having a cutoff distance (e.g., $12 \AA$ in a typical $\mathrm{C}_{\alpha}$ coarse-grained protein model). ${ }^{12-16}$ In contrast to ENM, MWCENM is a more precise 


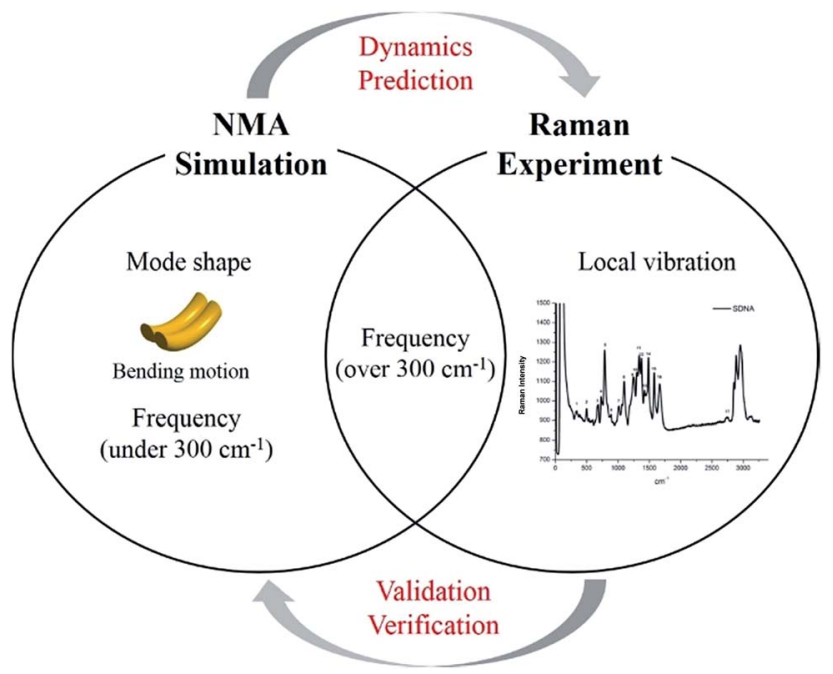

Fig. 1 Highlight of the proposed frequency analysis method. Raman spectrum experiment has been widely utilized as a tool for identification of nanostructure by means of its vibrational fingerprint within a limited frequency range (i.e., over $300 \mathrm{~cm}^{-1}$ ). However, this experimental technique cannot capture structural features and dynamics, which are the most important factors to be understood at atomic details because their functions are mainly determined by their lowfrequency vibrations (i.e., collective motions). On the other hand, the proposed MWCENM based NMA simulation enables us to calculate all the vibration frequencies and their corresponding mode shapes from collective motions to local vibrations. By direct comparison of vibration frequencies between Raman and NMA, one can assure that lowfrequency dynamics of DNA nanostructures can be predicted by simulation at atomic details.

Table 1 Various spring constants given by the type of chemical bond. In the MWCENM, van der Waals, hydrogen, ion, disulfide, and covalent bonds are assigned different spring constants. The spring constant ratio of van der Waals, hydrogen, and covalent bonds is $1: 10: 100$

\begin{tabular}{ll}
\hline Chemical bond & Spring constant $\left[\mathrm{N} \mathrm{m}^{-1}\right]$ \\
\hline van der Waals bond & 7 \\
Hydrogen bond & 70 \\
Ion bond & 70 \\
Disulfide bond & 70 \\
Covalent bond & 700
\end{tabular}

modeling method because it considers both the inertia effect and chemical bond information of the target system. The inertia effect is given by lumped masses to representative atoms of the target system, and chemical bonds are considered by assigning different spring constants, as shown in Table $1 .^{17,18}$ Therefore, both the frequencies and mode shapes of the target system can be obtained using the MWCENM. This method was already validated for both protein and DNA dynamics simulation elsewhere..$^{17,20}$

\section{Normal mode analysis (NMA)}

We performed MWCENM-based NMA to calculate the frequencies of two different DX DNA tiles. Suppose there is a system of $N$ particles. Each particle is a representative atom chosen through sampling and a lumped mass that includes the weight of surrounding atoms. We construct a spring network between particles using the chemical bond types (Table 1) and then construct the equation of motion for the system as follows. The position of the $i^{\text {th }}$ atom at time $t, x_{i}(t)$, can be represented by the initial position $x_{i}(0)$ and a small displacement $\delta(t)$ so that $x_{i}(t)=x_{i}(0)+\delta(t)$ where $x_{i}(t)$ is a $3 \times 1$ vector. Then, the kinetic energy of the system can be defined as

$$
T=\frac{1}{2} \sum_{i=1}^{n} m_{i}\left\|\dot{x_{i}}(t)\right\|^{2},
$$

where $m_{i}$ is the weight of a specific lumped mass. The kinetic energy can be rewritten in matrix form as

$$
T=\frac{1}{2} \dot{\delta}^{T} M \dot{\delta},
$$

where $M$ is a global mass matrix consisting of the lumped masses and $\delta$ is a $3 N \times 1$ matrix. In addition, the potential energy can be written as

$$
V=\frac{1}{2} \sum_{i=1}^{n-1} \sum_{j=i+1}^{n} k_{i, j}\left\{\left\|x_{i}(0)-x_{j}(0)\right\|-\left\|\delta_{i}(t)-\delta_{j}(t)\right\|\right\}^{2},
$$

where $k_{i, j}$ is the spring constant between the $i^{\text {th }}$ and $j^{\text {th }}$ atoms. By using a Taylor series approximation, we can rewrite eqn (3) as

$$
\begin{aligned}
V= & \frac{1}{2} \sum_{i=1}^{n-1} \sum_{j=i+1}^{n} k_{i, j}\left(\delta_{i}(t)-\delta_{j}(t)\right)^{T} \\
& \times\left(\frac{\left(x_{i}(0)-x_{j}(0)\right)\left(x_{i}(0)-x_{j}(0)\right)^{T}}{\left\|x_{i}(0)-x_{j}(0)\right\|}\right)\left(\delta_{i}(t)-\delta_{j}(t)\right) .
\end{aligned}
$$

Then, we can derive the equation of motion using Lagrange's equation.

$$
\frac{\mathrm{d}}{\mathrm{d} t}\left(\frac{\partial L}{\partial \dot{\delta}_{i}}\right)-\frac{\partial L}{\partial \delta_{i}}=0
$$

Here, $L=T-V$. Therefore, the equation of motion for the system is

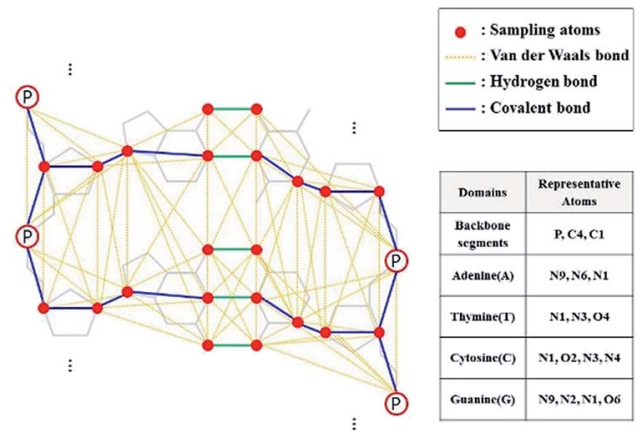

Fig. 2 DNA sampling model and its representative atoms. Three or four atoms were chosen to represent the atoms at each domain, and they are colored red. van der Waals, hydrogen, and covalent bonds are set by cutoff distance $(8 \AA)$, coordinates of DNA structure, and DNA linkages (such as glycosidic and ester links), respectively. 


$$
M \ddot{\delta}+K \delta=0
$$

By solving eqn (6), we can obtain both the vibrational frequencies and corresponding vibration mode from the eigenvalues and eigenvector, respectively. ${ }^{19}$

\section{DNA sampling model}

In this study, we conducted DNA sampling as shown in Fig. 2. In the sugar-phosphate backbone part, P, C1, and C4 atoms were selected. In each base part, we chose 3 or 4 atoms related to the backbone and hydrogen bonds between base pairs.

\section{Frequency calculation}

If there is an eigenvalue $\lambda$ from the solution to eqn (6), the frequency calculation is written as follows:

$$
f=\frac{1}{2 \pi} \sqrt{\lambda}
$$

where the unit of $f$ is Hz. The unit for the Raman spectroscopy data is $\mathrm{cm}^{-1}$, so we converted the unit of frequency to $\mathrm{cm}^{-1}$ according to

$$
f^{\prime}=\frac{f}{100 c},
$$

where $c$ is the speed of the light.

\section{Raman spectrum measurement}

We performed the Raman measurements of DNA molecules at room temperature with a confocal Raman microscope (WITEC, alpha $300 \mathrm{R}$ ) at $532 \mathrm{~nm}$. The spectral reading was obtained using a 600 gr per $\mathrm{mm}$ diffraction grating with the height for each blade at $500 \mathrm{~nm}$. Due to the different atomic arrangements in the molecules, vibrational spectra obtained from Raman spectroscopy provide intrinsic characteristics of the molecules. To prevent the buffer effect during measurement of the Raman spectrum, we used pristine duplex DNA molecules extracted from salmon.

\section{Results and discussion}

The design scheme for DX DNA tiles and representative atomic force microscope (AFM) images of DX DNA lattices composed of DX tiles are shown in Fig. 3. A DX tile with the dimension of 12.6 $\times 4.0 \mathrm{~nm}^{2}$ is made of two duplexes connected by two crossover junctions. Two types of DX tiles-DX0 (no hairpin) and DX1 (with a single full turn-length duplex hairpin protruding upward with respect to the surface of the DX tile)-are used to test the frequency analysis method based on MWCENM (more detailed information about sample preparation and AFM imaging is in experimental section and Fig. S1 of the ESI $\dagger) .{ }^{20}$ In addition, we introduce DX0C (DX1C) to verify the structural stability of DX0 (DX1) lattices, which are composed of DX0 and DX0C (DX1 and DX1C) tiles. The vibrational characteristics of DX0 lattice (consisted of four DX0 tiles) are also obtained from the frequency analysis method and compared with Raman experimental data.

\section{Raman experimental data}

Table 2 shows the experimentally obtained Raman spectra and vibrational mode assignments of DNA molecules in the range between $400 \mathrm{~cm}^{-1}$ and $1200 \mathrm{~cm}^{-1}$ (Raman spectra and band assignments in the full range are shown in Fig. S3 and Table S1 of ESI $\dagger$ ). The significant Raman spectra of DNA nucleotides are categorized into two regions: $400-750 \mathrm{~cm}^{-1}$ for nucleobases and 750-1200 $\mathrm{cm}^{-1}$ for sugar phosphate backbone groups. ${ }^{21,22}$ Due to a certain limitation when taking Raman spectra through the synthetic DX tiles-exceeding the Raman signal of the $1 \times \mathrm{TAE} /$ $\mathrm{Mg}^{2+}$ buffer (40 $\mathrm{mM}$ Tris base, $20 \mathrm{mM}$ acetic acid, $1 \mathrm{mM}$ EDTA (pH 8.0), and $12.5 \mathrm{mM}$ magnesium acetate) rather than the signal of the DNA molecules - we alternatively obtained them from the natural DNA duplexes extracted from salmon, which are also valid because the frequency range compared with the simulation is higher than $300 \mathrm{~cm}^{-1}$. In that relatively high frequency range, the DNA molecule generally shows the local vibrational characteristics of backbones and specific bases.

\section{Frequency analysis data}

The MWCENM based frequency analysis simulation was performed to calculate the frequencies of individual DX tiles (i.e., DX0 and DX1). Here the fixed boundary condition was applied in order to reflect the experimental set up such as DNA duplex

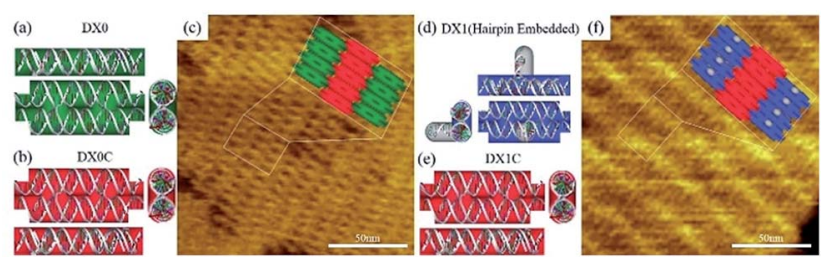

Fig. 3 Schematics of double crossover (DX) DNA building blocks and representative AFM images of DX lattices. (a, b) A DXO (green) is a planar DX building block without a hairpin, and a DXOC (red) connects DXOs. (c) A representative AFM image of a DX lattice comprised of DXO and DXOC tiles. (d, e) A DX1 (blue) is a hairpinembedded DX building block, and a DX1C connects DX1s. (f) An AFM image of a DX lattice composed of DX1 and DX1C tiles. The scan size of the AFM images is $150 \times 150 \mathrm{~nm}^{2}$.

Table 2 Raman spectrum data for pristine DNA molecules obtained from salmon. Seven representative Raman spectral peaks in the range between $400 \mathrm{~cm}^{-1}$ and $1200 \mathrm{~cm}^{-1}$ are displayed to validate the

\begin{tabular}{|c|c|c|}
\hline No. & $\begin{array}{l}\text { Raman shift } \\
\left(\mathrm{cm}^{-1}\right)\end{array}$ & DNA vibrational modes \\
\hline 1 & 497 & Phosphoionic scissor \\
\hline 2 & 678 & Ring breathing vibrations of $\mathrm{G}$ and $\mathrm{T}$ \\
\hline 3 & 732 & Ring breathing vibrations of $\mathrm{A}$ \\
\hline 4 & 786 & $\begin{array}{l}\text { Vibrations of } \mathrm{C}, \mathrm{T} \text {, and } \\
\text { symmetric stretching of } \mathrm{PO}_{2}^{-}\end{array}$ \\
\hline 5 & 890 & Deoxyribose ring vibrations \\
\hline 6 & 1012 & $\mathrm{C}-\mathrm{O}$ stretching vibrations of deoxyribose \\
\hline 7 & 1095 & Phosphodiester stretching vibrations \\
\hline
\end{tabular}
calculated frequencies (Raman spectra in the full range are shown in Table S1 of ESI) 


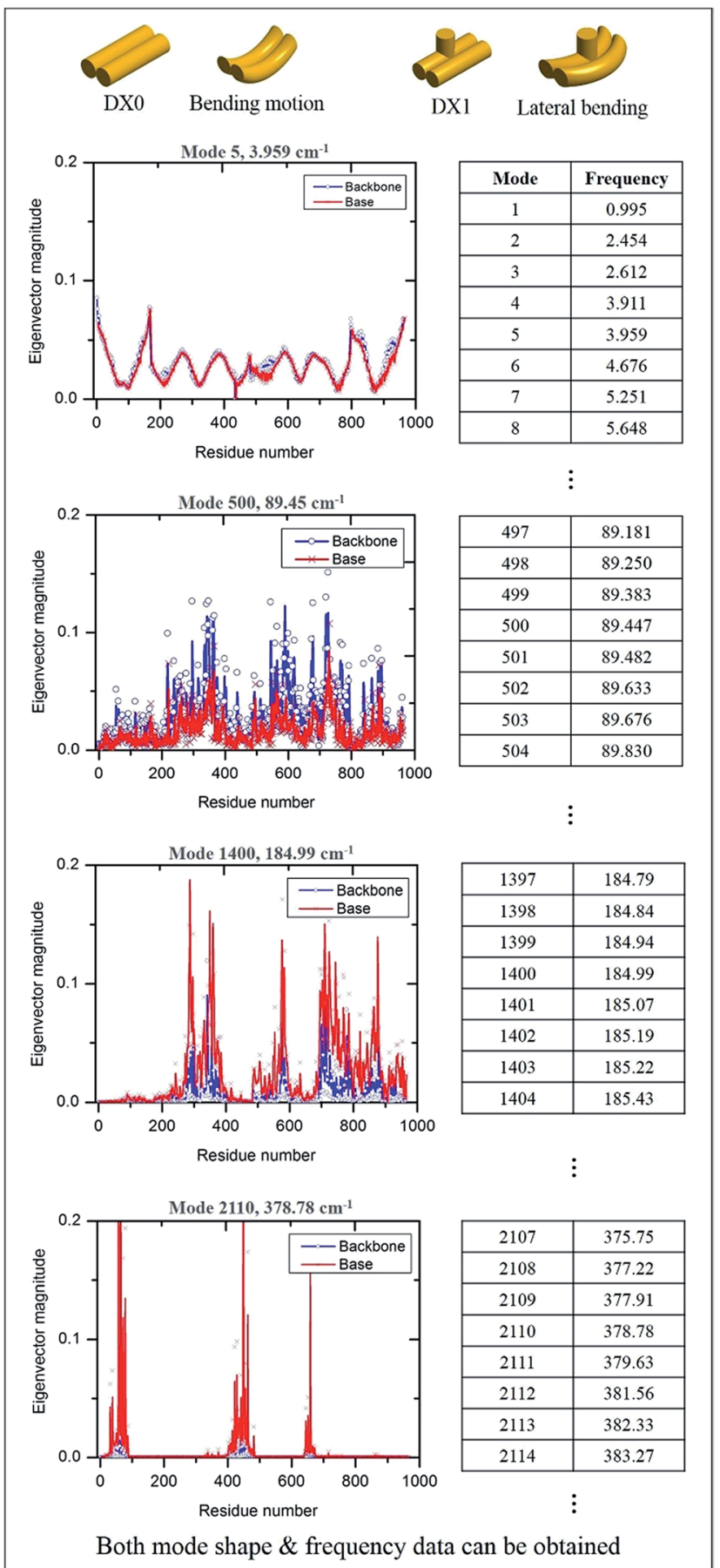

Fig. 4 Vibrational properties of DXO and DX1 and their frequencies. By solving the equation of motion and using frequency calculations, we obtained both vibrational mode shapes and their corresponding frequencies. The number of modes depends on the number of sample atoms, and the vibrational characteristics from low to high frequency show dynamic motion, from collective motion to local vibration. Four vibrational characteristic graphs are obtained from the equation of motion of DXO (the characteristics of DX1 are not shown here because they are similar to those of DXO).

fixed on a substrate (refer to simulation section of ESI $\dagger$ ). By solving eqn (6), we obtained the eigenvalues at each vibrational mode of DX0 and DX1, and then we calculated the frequencies
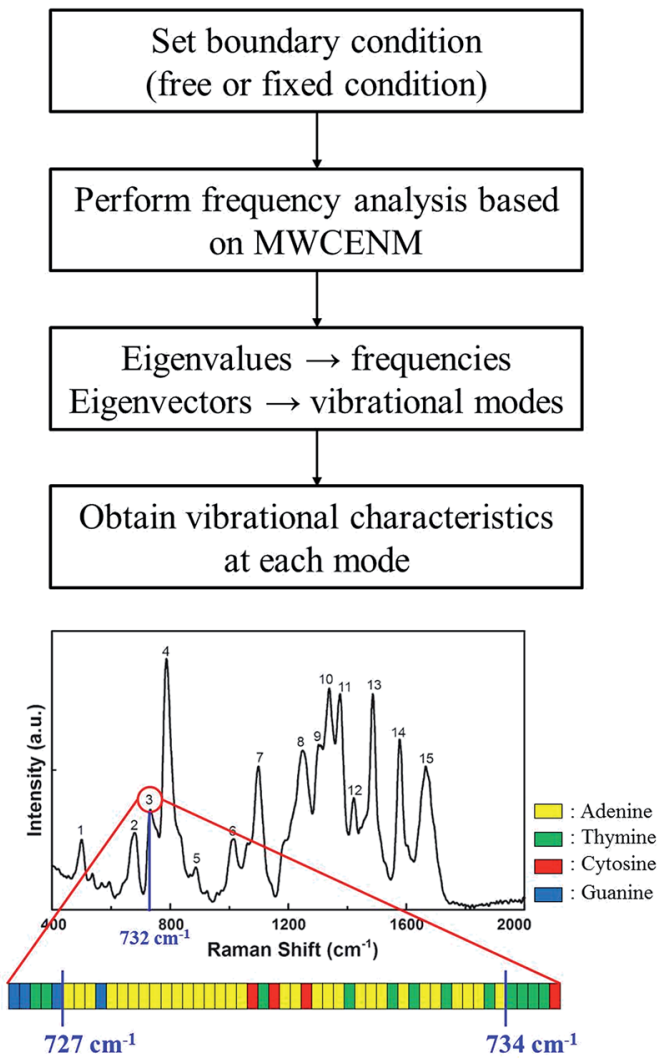

Comparison between Raman spectrum and simulation

Fig. 5 Identification procedure of base type in the specific region part. In case of DXO at fixed boundary condition, a total of 2823 different normal modes including both frequencies and vibrational mode shapes were obtained and classified into 4 parts as shown in Fig. 4. In the specific region part, there were particularly local vibrations in which only a few atoms show high mobility in specific bases. Systematically, if their eigenvector magnitudes are larger than 0.1, we considered them to have high mobility and they were sorted by their base types. Finally, such base type and corresponding frequency information were compared with Raman experimental data. For example, corresponding to the third Raman peak $\left(732 \mathrm{~cm}^{-1}\right)$ which is known as a ring breathing motion of adenine, the MWCENM simulation successfully identified high mobility in adenine $\left(727-734 \mathrm{~cm}^{-1}\right)$. Although a few other bases also appeared in this frequency range, adenine is the most dominant and followed by thymine as its complementary base.

at each mode by solving eqn (7) and (8) in sequence. We found that the main modes of DX0 are bending and twisting, like general rod motions, whereas DX1 bends and twists with a hairpin bending motion (vibrational mode shapes of DX0 and DX1 are both available in Fig. S2 of ESI $\dagger$ ). As frequency increases, in both tile structures, the overall vibrational characteristics evolve from global collective motions such as bending and twisting to local molecular vibrations as demonstrated in Fig. 4.

For instance, in case of DX0 at fixed condition, its frequencies are divided into four parts such as the collective part (9.5$\left.21.6 \mathrm{~cm}^{-1}\right)$, phosphate backbone part $\left(22.1-150.9 \mathrm{~cm}^{-1}\right)$, base part $\left(151.0-265.0 \mathrm{~cm}^{-1}\right)$, and specific region part (265.2$\left.1166.9 \mathrm{~cm}^{-1}\right)$. Likewise, the other simulation cases are also 
summarized in Table S2 of ESI. $\dagger$ The collective part exhibits collective motions of both phosphate backbone and base to the same direction. However, the phosphate backbone part (the base part) has high mobility only at phosphate backbone (base). Furthermore, the specific region part shows high mobility in some local bases. We systematically counted the number of occurrence of high mobility in each base when its magnitude of eigenvector is higher than 0.1. This number counting yields automatic classification of the frequency ranges, in each of which only the same base types dominantly show high mobility. Through this procedure, specific frequency ranges were confirmed to each corresponding Raman peak. For instance, as shown in Fig. 5, the frequency range of DX0 at fixed condition that shows high mobility in adenine is $727-734 \mathrm{~cm}^{-1}$. This simulation result matches well with the third Raman peak (732 $\mathrm{cm}^{-1}$ ) which is known as a ring breathing motion of adenine.

Moreover, in order to investigate the boundary condition effect, we additionally performed simulation at free boundary condition for both DX0 and DX1. It is noted that the first six zero eigenvalues, in the standard mathematical framework of NMA, are to be excluded because they correspond to rigid body motions (i.e., three translational motions and three rotational motions) of the given system at free boundary condition.

In our comparison between the simulation frequencies of DX0 (DX1) and the experimental Raman data, shown in Fig. 6a, b (c, d), the percentages of error deviations (defined as $100 \times$ |Raman data - simulation data|/Raman data) are relatively smaller at fixed condition, $1.9 \%(1.6 \%)$, compared to free condition, $4.0 \%(2.0 \%)$. Although small error deviations are observable, the overall trends between simulation and experiment are similar for both free and fixed conditions. Therefore, we not only validated the proposed frequency analysis method based on MWCENM, but also demonstrated the reliability of comparison between predicted frequency data and Raman experimental data. After successful validation of modelling and simulation using a single tile structure, furthermore, we investigated the vibrational characteristics of DX0 lattice structure at fixed condition. As shown in Fig. 6e, it has similar vibrational features of DX0 (DX1) single tile but the error with Raman data dramatically reduces down to $0.7 \%$. Although here we only conducted the validation of the proposed frequency analysis method at the high frequency range due to experimental limits, it implies that the MWCENM simulation can predict vibrational

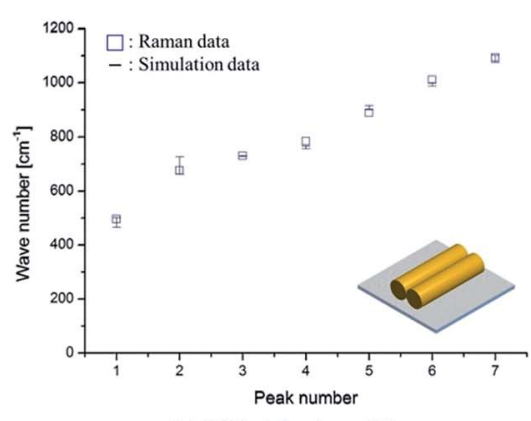

(a) DX0 at fixed condition

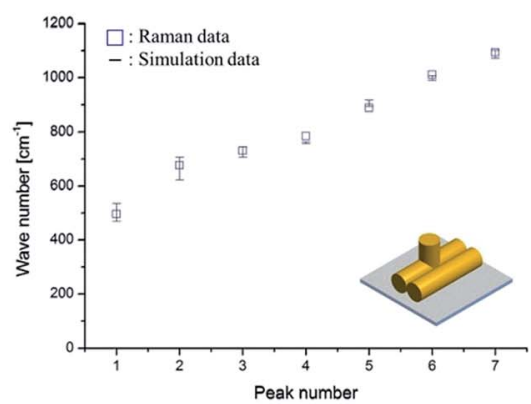

(c) DX1 at fixed condition

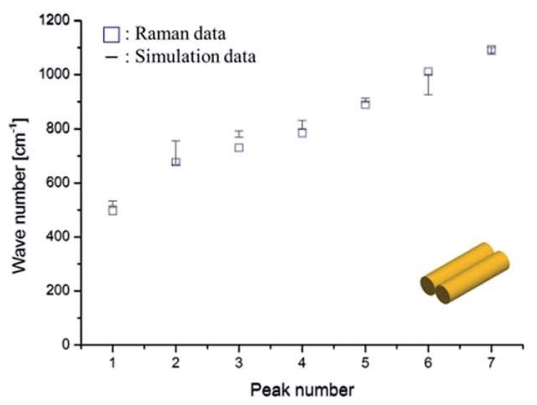

(b) DX0 at free condition

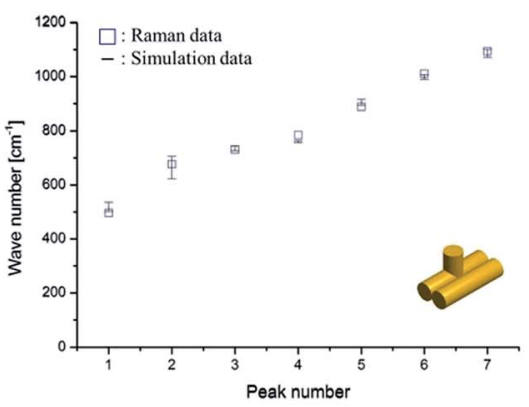

(d) DX1 at free condition

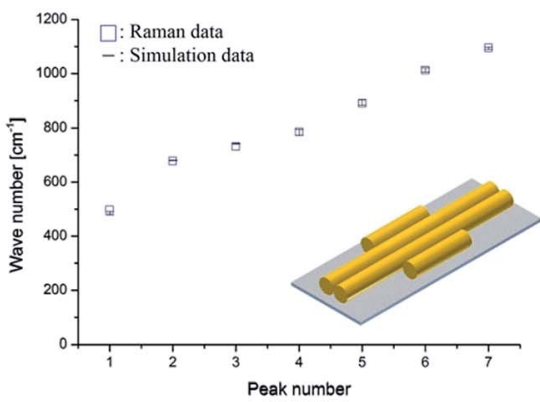

(e) DX0 lattice at fixed condition

Fig. 6 Vibrational frequency comparison between Raman spectra and the MWCENM simulation. (a, b) Vibrational frequencies (squares for experimentally obtained Raman data and bars for theoretically computed frequency ranges) of DXO at fixed (bound on the substrate) and free conditions. The computed frequency ranges of DXO in the fixed (free) condition are 463-501, 660-726, 727-734, 755-766, 897-914, 9861000 , and $1074-1100 \mathrm{~cm}^{-1}\left(514-532,668-755,769-792,800-830,896-911,924-1000\right.$, and $\left.1074-1100 \mathrm{~cm}^{-1}\right)$ as the peak number increases from 1 to 7 , respectively. Although the free and fixed conditions show similar trends, the error (difference between experimental values and the median of the computed frequency range) in the fixed condition is relatively smaller than the error in the free condition because of better consideration of the experimental setup. The average error decreased from $4.0 \%$ (free condition) to $1.9 \%$ (fixed). (c, d) Vibrational frequencies of DX1 at fixed and free conditions. The computed frequency ranges of DX1 in the fixed (free) condition are 467-535, 621-705, 706-745, 756-763, $892-915,988-1006$, and $1070-1100 \mathrm{~cm}^{-1}\left(500-535,621-705,725-745,756-763,892-915,988-1006\right.$, and 1070-1100 cm $\left.{ }^{-1}\right)$. The average error decreases from $2.0 \%$ (free condition) to $1.6 \%$ (fixed). (e) Vibrational frequencies of DX0 lattice at fixed condition. The computed frequency ranges are 479-493, 678-682, 741-744, 773-795, 883-903, 1007-1021, and 1090-1098 $\mathrm{cm}^{-1}$. This simulation result shows the highest accuracy with an error of $0.7 \%$. 
features (i.e., both frequency and mode shape) of DNA tile structures very precisely and effectively in terms of accuracy and computational cost, respectively.

\section{Conclusions}

In this study, we compared frequencies calculated using the MWCENM method with Raman experimental data. In order to confirm the reliability of calculated frequencies and boundary effect, we considered both free and fixed boundary conditions in case of DX0 and DX1 single tiles. Moreover, the vibrational characteristics of DX0 lattice at fixed condition were also investigated and compared with Raman spectrum of DNA duplex. Due to experimental limits, we just compared our simulation result with Raman experimental data at the high frequency range. That is, only local vibrational characteristics of backbone and base of DNA molecules can be obtained through experiment, whereas simulation can capture the wide range of atomic motions from the low frequency (collective motion) to the high frequency (local vibration). For all cases, the proposed frequency analysis method successfully captured vibrational features of DNA tile structures, at most, with an error of $4.0 \%$. Consequently, it is expected that this study will play an important role in prediction of structural stability and functional dynamics of biomolecules at atomic level which are still hardly obtained through any state-of-the-art experimental method.

\section{Conflicts of interest}

There are no conflicts to declare.

\section{Acknowledgements}

This research was supported by the Basic Science Research Program through the National Research Foundation of Korea (NRF), funded by the Ministry of Education (2015R1D1A1A01057280, M. K. K.), (2014R1A2A1A11053213, S. H. P.).

\section{Notes and references}

1 N. C. Seeman, J. Theor. Biol., 1982, 99, 237-247.
2 E. Winfree, F. Liu, L. A. Wnzler and N. C. Seeman, Nature, 1998, 394, 539-544.

3 S. Hamada and S. Murata, Angew. Chem., Int. Ed., 2009, 48, 6820-6823.

4 P. Yin, R. F. Hariadi, S. Sahu, H. M. Choi, S. H. Park, T. H. Labean and J. H. Reif, Science, 2008, 321, 824-826.

5 D. Liu, M. Wang, Z. Deng, R. Walulu and C. Mao, J. Am. Chem. Soc., 2004, 126, 2324-2325.

6 P. W. K. Rothemund, Nature, 2006, 440, 297-302.

7 B. Wei, M. Dai and P. Yin, Nature, 2012, 485, 623-626.

8 N. C. Seeman, Annu. Rev. Biophys. Biomol. Struct., 1998, 27, 225-248.

9 A. V. Pinheiro, D. Han, W. M. Shih and H. Yan, Nat. Nanotechnol., 2011, 6, 763-772.

10 M. Zahid, B. Kim, R. Hussain, R. Amin and S. H. Park, Nanoscale Res. Lett., 2013, 8, 119.

11 P. F. Qian, S. Seo, J. Kim, S. Kim, B. S. Lim, W. K. Liu, B. J. Kim, T. H. LaBean, S. H. Park and M. K. Kim, Nanotechnology, 2012, 23, 105704.

12 A. D. Schuyler and G. S. Chirikjian, J. Mol. Graphics Modell., 2004, 22, 183-193.

13 V. Tozzini, Curr. Opin. Cell Biol., 2005, 15, 144-150.

14 P. Doruker, A. R. Atilgan and I. Bahar, Proteins, 2000, 40, 512-524.

15 A. R. Atilgan, S. R. Durell, R. L. Jernigan, M. C. Demirel, O. Keskin and I. Bahar, Biophys. J., 2001, 80, 505-515.

16 K. Hinsen, Proteins, 1998, 33, 417-429.

17 M. H. Kim, S. Seo, J. I. Jeong, B. J. Kim, W. K. Liu, B. S. Lim, J. B. Choi and M. K. Kim, Protein Sci., 2013, 22, 605-613.

18 M. Hu, S. Raj, B. Kim, W. K. Liu, S. Baik, T. Kim, B. S. Lim and M. K. Kim, J. Mech. Sci. Technol., 2010, 24, 1771-1780.

19 M. K. Kim, G. S. Chirikjian and R. L. Jernigan, J. Mol. Graphics Modell., 2002, 21, 151-160.

20 B. Kim, S. Jo, J. Son, J. Kim, M. H. Kim, S. U. Hwang, S. R. Dugasani, B. D. Kim, W. K. Liu, M. K. Kim and S. H. Park, Nanotechnology, 2014, 25, 105601.

21 A. Kulkarni, B. Kim, S. R. Dugasani, P. Joshirao, J. A. Kim, C. Vyas, V. Manchanda, T. Kim and S. H. Park, Sci. Rep., 2013, 3, 2062.

22 B. Gnapareddy, S. R. Dugasani, T. Ha, B. Paulson, T. Hwang, T. Kim, J. H. Kim, K. Oh and S. H. Park, Sci. Rep., 2015, 5, 12722 . 